\title{
Contribuciones teóricas para la construcción de epistemologías otras no androcéntricas: una aproximación al planteamiento feminista de Gloria Comesaña Santalices
}

Theoretical contributions for building other non-androcentric epistemologies: an approach to the feminist thinking of Gloria Comesaña Santalices

Contribuições teóricas para construção de epistemologia outras, não-androcêntricos: uma aproximação à abordagem feminista de Gloria Comesaña Santalices

María Laura Stephen-Chaves

Escuela de Filosofía

Universidad Nacional de Costa Rica

Recibido: 16/02/2015 -Aceptado: 26/6/2015

http://dx.doi.org/10.15359/tdna.31-58.5

Resumen

Pretendo identificar, mediante el estudio de algunos de sus textos, las principales contribuciones teóricas que ha realizado la pensadora Gloria Comesaña Santalices para el proceso de construcción de una/s epistemología/s feminista/s, mediante el acercamiento a propuestas alternativas a las

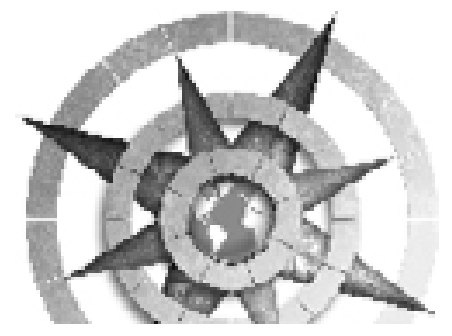

epistemologías androcéntricas que se han constituido como dominantes a lo largo de la historia. Asimismo, haré referencia a aspectos claves de la epistemología feminista que plantea la autora Comesaña Santalices, mediante un recorrido por la terminología con que desarrolla sus planteamientos

Palabras claves: Gloria Comesaña Santalices, pensamiento feminista latinoamericano, epistemología feminista, decolonialidad, Temas de Nuestra América 


\begin{abstract}
By studying some of Comesaña Santalices' texts, I intend to identify the main theoretical contributions made by this thinker in her process of building a feminist epistemology as alternative proposals to the historically dominant androcentric epistemology. I will also refer to key aspects of Comesaña Santalices feminist epistemology by analyzing the terminology which she develops her theoretical approaches.
\end{abstract}

Keywords: Gloria Comesaña Santalices, feminist Latin American thinking, feminist epistemology, decolonialism, Temas de Nuestra América

\section{Resumo}

Através do estudo de alguns de seus textos, eu tento identificar as principais contribuições teóricas feitas pela pensadora Gloria Comesaña Santalices para a construção de uma epistemologia feminista trazendo propostas alternativas para a epistemologias androcêntricas que se tornaram tão dominantes ao longo da história. Além disso, vou me referir a aspectos-chave da epistemologia feminista levantada pela autora Comesaña Santalices analisando a terminologia com que ela desenvolve suas abordagens.

Palavras chave: Gloria Comesaña Santalices, pensamiento feminista latinoamericano, epistemología feminista, decolonialidade, Temas de Nuestra América
He de iniciar este desarrollo señalando que en el presente escrito "No pretendemos ser exhaustivas, ni en cuanto a los temas ni al manejo de los mismos, sino presentar algunas líneas de reflexión que nos parecen prioritarias" (Comesaña, 2007, p. 99).

Es pertinente realizar algunas aclaraciones previas antes de ahondar en los planteamientos de Gloria Comesaña Santalices, esto para lograr una mayor comprensión de los mismos y valorar su importancia. Considero que para entrar a abordar el tema de la epistemología feminista, es preciso tener claros conceptos como epistemología y feminismo. A continuación intentaré una aproximación a dichos términos con fines de facilitamiento pedagógico.

El término epistemología viene del griego; de episteme, que significa conocimiento y de logia (conformada por la palabra logos) que significa estudio. Episteme hace referencia al conocimiento verdadero en contraste con la doxa que alude a las creencias, a la mera opinión que no alcanza a ser considerada como conocimiento legítimo por no haber sido sometido a una rigurosa reflexión crítica.

Etimológicamente significa estudio del conocimiento. A grandes rasgos,

92 Contribuciones teóricas para la construcción de epistemologías otras no androcéntricas: una aproximación al planteamiento feminista de Gloria Comesaña Santalices María Laura Stephen-Chaves 
cabe señalar, que la epistemología es esa rama de la filosofía que se encarga de estudiar los problemas filosóficos que se dan en torno al conocimiento y lo que esto implica, es decir, abarca cuestionamientos como ¿qué es el conocimiento?, ¿cómo conocemos?, ¿cuál es la relación entre el sujeto cognoscente y lo que pretende conocer?, ¿cómo se genera y cómo se valida el conocimiento?, ¿cuáles son los límites de este?...

En síntesis, esta rama de la filosofía trata aspectos como el método a seguir para alcanzar el conocimiento, la relación sujeto-objeto o sujeto-sujeto, abordaje de conceptos como verdad-falsedad, objetividad... trata "de ver cuáles son las posibilidades y los límites del conocer humano en el tratamiento de ésta o aquella cuestión" (González, 1989, p. 37). Más adelante retomaré este tema.

Respecto al feminismo, mencionaré una serie de citas de diversas autoras que han intentado definir este término, para posteriormente recopilar los aspectos claves que cada una de ellas menciona y de esta forma lograr una mayor comprensión de eso que hemos llamado feminismo. Veamos seguidamente el análisis que hacen tres conocidas autoras:
Ser feminista es descubrir la realidad de la condición subordinada y oprimida en que viven las mujeres consideradas en cuanto grupo humano. Es reconocer que dicha condición no se explica ni por la Naturaleza ni por cualquiera otra fuerza superior, sino que es el resultado de una cierta organización social producida por una Historia que puede y debe descubrirse y desenmascararse (Comesaña, 1995, p. 89).

El feminismo es una corriente política de la modernidad que ha cruzado la historia contemporánea desde la Revolución Francesa hasta nuestros días, aunque tiene antecedentes que pueden rastrearse en los escritos de la Edad Media y el Renacimiento (Gargallo, 1997, p.5).

El feminismo como tal comienza a gestarse ideológicamente durante la Ilustración y se concreta en los albores de la Revolución Francesa. El feminismo tiene sus orígenes en la Modernidad; por ello la filósofa española Cristina Molina Petit sostiene que el feminismo es en principio una conquista ilustrada; y como tal hace sus primeras reivindicaciones teóricas en nombre de la universalidad de la razón. En esa época se dieron los primeros intentos de las mujeres por defender sus derechos; se organizaron masivamente en busca de la igualdad política con 
respecto al hombre, formando la nueva idea de la universalidad de la razón como argumento (Rincón, 2009, pp. 23-24).

De las citas anteriores destacan aspecto destaco aspectos tan fundamentales como que el feminismo es un movimiento o una corriente revolucionaria que tiene sus inicios en la Modernidad, problematiza la condición de subordinación a la que se ha relegado a la mujer, a la vez que emprende luchas por la igualdad de condiciones y la emancipación de las mismas. Además de ello implementa la visión histórico-social para el análisis de las problemáticas que han aquejado y aquejan tanto a hombres como mujeres, con ello visibilizan que estas se deben a constructos sociales que se han dado en determinados contextos y que por ende no son absolutos ni estáticos sino pueden modificarse.

El feminismo como movimiento y corriente histórica ha estado en constante cambio, regenerándose a partir de las experiencias vividas, de las luchas alcanzadas y las que quedan por alcanzar. Desde sus inicios ha tenidos múltiples cambios que han derivado en nuevos enfoques, por ejemplo según Comesaña (1995) durante los años sesenta tiene cabida el feminismo contemporáneo, mismo que venía gestándose desde finales del siglo XIX y a inicios del XX. En este período se emprenden las luchas sufragistas. Durante los años setentas empiezan a surgir nuevos proyectos sociales y políticos como los autodenominados feminismos negros y los indígenas, que lanzan fuertes críticas hacia el feminismo emergente, ya que este tiene claros matices occidentalocéntricos, racistas, burgueses, elitistas, colonialistas y heteronormativos. Esto por mencionar algunos ejemplos, ya que existen muchos más.

Como último aspecto considero importante tener presente que si bien, en los aspectos formales el feminismo surge a finales del siglo XVIII, esta corriente, retomando las palabras de Comesaña (1995) y los planteamientos de diversas feministas negras, se remonta mucho antes de esta época si consideramos como feminista a toda aquella persona que se haya preocupado por concientizar sobre la condición de las mujeres, la opresión a las que han sido sometidas y además de esto luche por transformar esta situación.

Ahora bien, una vez aclarados los conceptos anteriores retomaré el tema de la epistemología. Si bien, como mencioné anteriormente, a grandes rasgos la epistemología es

94 Contribuciones teóricas para la construcción de epistemologías otras no androcéntricas: una aproximación al planteamiento feminista de Gloria Comesaña Santalices María Laura Stephen-Chaves 
esa rama de la filosofía que se encarga del estudio del conocimiento, a lo largo de la historia no todo saber ha sido reconocido como conocimiento verdadero y no a todos los seres humanos se les ha considerado como sujetos capaces de desarrollar, emprender o alcanzar ese conocimiento acerca de las cosas o del entorno en que se encuentran inmersos/as. Incluso diversos postulados o teorías que han formulado diferentes sujetos para explicar determinados fenómenos son tachados como inválidos per se, dada su cultura, grupo étnico al que pertenecen, su sexo, género, lugar de origen... Es decir, ese "conocimiento" del que se encarga la epistemología se ha encontrado y se encuentra restringido a parámetros sumamente rígidos.

A lo largo de las diferentes épocas históricas determinadas formas de pensar, de abordar, analizar los problemas, determinadas prácticas se han constituido como hegemónicas. En ello han intervenido diversos factores políticos, sociales, económicos, ideológicos... que han confluido en determinado contexto y han contribuido a que ciertos aspectos hayan sido considerados o impuestos como superiores a otros existentes. En este caso la ciencia, el método científico y la figura del hombre-masculino, han sido algunos de los parámetros que han condicionado el desarrollo del conocimiento que se ha dado durante el transcurso de la historia.

En cuanto al primer parámetro, podría afirmar que desde la Antigua Grecia hasta la actualidad, tanto la ciencia como el conocimiento científico han sido valorados de forma positiva. Claro que lo que se entiende por ciencia ha variado en las diferentes épocas históricas, es decir, el concepto de ciencia y el método científico actual no es el mismo que utilizaban los presocráticos u otros filósofos/ as en otras épocas precedentes. Sin embargo, aunque su significado y método hayan variado, la superioridad que se les ha otorgado se ha mantenido hasta la actualidad, quizás con excepción de la Edad Media. Incluso la misma palabra episteme se suele traducir como ciencia o conocimiento científico, ya que, desde el paradigma dominante la palabra conocimiento o conocimiento verdadero y ciencia o conocimiento científico, funcionan como equivalentes.

Diversas fuentes consultadas, para la elaboración del presente escrito, definían epistemología como el estudio de la ciencia o del conocimiento científico, sin embargo, en el presente ensayo no he utilizado esta definición debido a que desde mi punto de vista el estudio de la ciencia, en 
específico, concierne más a la filosofía de la ciencia que a la epistemología, sería un error considerar que solo desde la ciencia se ha producido conocimiento. Ante esto no estoy de acuerdo con utilizar como equivalentes conocimiento y ciencia, porque desde esta visión se han negado diversos conocimientos que por el hecho de no ser categorizados como científicos se les ha desprovisto de toda validez. Considero fundamental, como proyecto filosófico-epistemológico y político, replantear lo que comprendemos como episteme y su contraparte doxa. Cabe señalar que

El tema del conocimiento está íntimamente ligado a otras cuestiones filosóficas: en realidad, no se puede hablar sobre el conocimiento sin hablar sobre el hombre que conoce. Igualmente, no es posible hacerse una idea sobre el conocimiento humano, sin concebir de algún modo la realidad que es conocida (González, 1989, p. 37).

En esta cita, González menciona dos aspectos fundamentales para comprender el segundo parámetro que, desde mi punto de vista, ha condicionado el desarrollo del conocimiento; es decir, la visión y figura del hombre-masculino. El primer aspecto a tomar en cuenta es que no se puede hablar sobre el conocimiento, sin hablar del hombre que conoce. Esto es un aspecto filosófico básico, ya que, los sistemas de conocimiento dominantes, legitimados por la historia oficial, han sido emprendidos por un hombre que conoce, es decir, el sujeto cognoscente de esta realidad ansiosa de ser estudiada ha sido un sujeto con características que se han categorizado como masculinas.

¡Pero han existido mujeres que han planteado diversas teorías! Podrían argüir por allí. Claro que las ha habido pero no han sido reconocidas por la historia oficial. Esto porque a partir de que el hombre, masculino se constituyó como la figura dominante, como la noción de lo universal y la parte legitimada de la humanidad, la mujer fue excluida de diversos ámbitos entre ellos los del conocimiento, la ciencia, la política, la vida pública, quedando relegada al espacio privado como fiel subordinada del hombre.

El segundo aspecto al que hace referencia González (1989) es que no es posible hacerse una idea sobre el conocimiento humano, sin concebir de algún modo la realidad que es conocida. Esta realidad o estas realidades se encuentran marcadas por diversos sistemas histórico-sociales, entre ellos el patriarcado, por lo que de este modo podría decir que el conocimiento de

96 Contribuciones teóricas para la construcción de epistemologías otras no androcéntricas: una aproximación al planteamiento feminista de Gloria Comesaña Santalices María Laura Stephen-Chaves 
dicha realidad o realidades se va a encontrar mediados por el contexto en el que se desarrolle.

Sin duda, producto del sistema patriarcal, las mujeres han sido excluidas como sujetos activos de la historia que contribuyen en la producción de conocimientos. De esta forma la mayoría de aportes hechos desde las mujeres han sido relegados al olvido histórico, borrándose de esta forma su legado y su participación en la historia de la humanidad. De esto rinden cuenta diversas autoras, entre ellas, Francesca Gargallo quien en su trabajo intitulado Las ideas feministas latinoamericanas (1997) explica cómo se le ha negado a la mujer el acceso a lo universal y cómo se le ha sometido a un mundo regido por la racionalidad masculina. Esto debido a que el hombre se ha constituido como la figura universal en el parámetro de lo humano, de lo legítimo, a partir de esto ha intentado ubicar todo lo que le rodea dentro de sus categorías. Dentro de este mundo falocrático o patriarcal la mujer ha sido categorizada como inferior.

La siguiente cita muestra un resumen de estas ideas "sentimos en la piel qué significan los siglos durante los cuales ser humano se dijo hombre y lo universal se identificó con lo masculino" (Gargallo, 1997, p. 5).
La autora muestra a partir de diversos ejemplos como se le ha negado a la mujer participar dentro de la esfera de lo público, sus derechos políticos y como se le ha ridiculizado por emprender ceñidas luchas para librarse de la opresión.

Un claro ejemplo es la Revolución Francesa donde se les negó a las mujeres la participación en la esfera de lo público, en la política, bajo el argumento de los supuestos roles naturales correspondientes a cada sexo. De esta forma queda constatado como las luchas reivindicativas por la igualdad, la fraternidad y la libertad, solamente estaban contempladas para un sector de la población, mismo que debía cumplir con las siguientes características: ser blancos y portar un pene como órgano genital.

En la misma línea Lucía Rincón Soto en su libro Bases histórico-filosóficas de la teoría feminista (2009) explica cómo a pesar de que durante la Ilustración se abogaba por la razón universal, esta solamente estaba pensada para los hombres: “(...) la Ilustración no cumple
sus promesas: la razón no es la
razón universal. La mujer en el
siglo de las luces, sigue siendo
definida como la Pasión, la Natu-
raleza, el refugio fantasmagórico
de lo originario previo al ámbito to originario previo al ámbito 
propiamente humano de lo social-civil". (Molina, 1994, p. 20 citada por Rincón, 2009, p. 28).

Vera Yamuni, en su texto, El ser y el valer de la mujer comparados con el ser y el valer del hombre, muestra, recurriendo a diversos discursos emitidos por algunos filósofos, como el ser y el valer de la mujer se ha concebido como inferior en comparación con el ser y el valer del hombre. Muestra como el ser de la mujer ha sido definido a partir del parámetro masculino y como a partir de ello se le han restringido campos de acción, como la intelectualidad, la política, la esfera pública, el poder expresar su sexualidad. En correlato con lo anterior cabe señalar que:

Legisladores, hombres religiosos, filósofos, escritores, sabios se han empeñado en demostrar que la condición subordinada de la mujer era querida por el cielo y provechosa en la tierra. La humanidad fue, y es todavía, masculina, y el hombre no ha considerado a la mujer como un ser autónomo, no la ha definido en sí sino relativamente a él; como él ha querido y decidido que sea. Ella se determina respecto y se diferencia con respecto al hombre, y no éste con respecto a ella; es lo inesencial frente a lo esencial. Él es el Sujeto, él es el Absoluto; ella es el Otro, lo subordinado, lo inferior.
(Beauvoir citada por Yamuni, 1985: p. 77).

Yadira Calvo, por su parte, con Éxtasis y ortigas. Las mujeres entre el goce y la censura (2004) evidencia como a lo largo de la historia a la mujer se le ha impedido el acceso al ámbito de la intelectualidad o como se han negado sus esfuerzos intelectuales por el mero hecho de su condición de mujer. Muestra como a partir del discurso de la complementariedad entre los sexos se impone que los hombres son aquella parte "inteligente, valerosa, esforzada, fuerte, valiente, hábil, razonable" mientras que su complemento, es decir, la mujer, es la parte "tonta, inútil, débil, sensible".

Además de ello, enuncia los argumentos que han utilizado diversos pensadores para justificar por qué la mujer no puede acceder al conocimiento, por qué este campo le está restringido, entre ellos se encuentran filósofos de peso que han tenido gran influencia. Según Calvo (2004) en un ambiente tan adverso, que les impedía a las mujeres acceder al estudio y el poder satisfacer su deseo de conocimiento, las estudiosas tuvieron que enfrentarse con una serie de obstáculos para lograr sus objetivos intelectuales. Sin duda, como lo menciona la autora, el que a la

98 Contribuciones teóricas para la construcción de epistemologías otras no androcéntricas: una aproximación al planteamiento feminista de Gloria Comesaña Santalices María Laura Stephen-Chaves 
mujer se le haya ligado con lo corporal y con lo irracional les ha impuesto grandes obstáculos para que las mismas puedan cumplir su deseo por la creación intelectual, además de que se han deslegitimado las producciones realizadas por ellas.

El caso de Sor Juana Inés de la Cruz, durante la segunda mitad del siglo XVII en México, es un claro ejemplo de cómo a las mujeres se les ha negado el acceso al ámbito del conocimiento y como se han desvalorizado las producciones y aportes intelectuales que las mismas han realizado. De Sor Juana, Yadira Calvo nos dice que:

"destacó por la versatilidad de su inteligencia: realizó experimentos científicos, compuso obras musicales; escribió una gran cantidad de poesías, varias obras dramáticas, un texto teológico titulado Carta Athenagórica en que se rebate un sermón del padre jesuita Vieyra, y la autobiografía Respuesta a Sor Filotea de la Cruz" (2004: p. 125).

Sin embargo, su producción no ha sido reconocida. Incluso dados los obstáculos sociales que se le interpusieron para poder satisfacer sus deseos de conocimiento, solo por su condición de mujer, Sor Juana decide recluirse en un convento de monjas. De este modo no se vería obligada a contraer matrimonio y podría continuar con sus estudios en los ratos libres. A pesar de que pudo librarse de ciertos condicionamientos sociales, no pudo escapar del todo. Sor Juana fue condenada por la Iglesia, ya que, a una mujer no le era permitido pensar, proponer, debatir, ahondarse en el mundo del conocimiento, debía relegarse a la sumisión y a la obediencia.

En síntesis, los aspectos que mencioné anteriormente me permiten evidenciar algunas de las características de la epistemología dominante. Para esta última el estudio del conocimiento implica el estudio de la ciencia o el conocimiento científico, mismo que ha sido construido por los hombres. La epistemología dominante responde al paradigma de la modernidad, que tiene su auge durante el siglo XVI. Época donde el conocimiento científico se torna como el único verdadero, superior al resto de los saberes. Dado esto, el método científico se impone como el único método a seguir para obtener conocimientos verídicos. Para ser categorizados como verdaderos deben ser claros, precisos, cuantificables, objetivos y universales (visión cartesiana). 
Desde el paradigma de la modernidad la ciencia tiene como función comprender el mundo o la naturaleza, para posteriormente dominarla, es decir, tiene como meta predecir y controlar las cosas, el entorno, con el fin de hallar lo que busca, arrancarle sus secretos (razón instrumental). Cabe agregar que la epistemología dominante tiene claros matices occidentalocéntricos.

A partir de la modernidad se desplaza a Dios como centro y "se desarrolla una cultura antropocéntrica, en la cual el Hombre a) es el centro del mundo y b) busca la gloria terrenal" (Rincón, 2009, p. 23). Con esto, el hombre se torna en la figura de lo universal, de la humanidad, como lo mencionaba Gargallo, en el único poseedor de la razón y por ende el único con la capacidad de elaborar conocimientos y teorías válidas que permitan explicar los diversos fenómenos que acontecen en el mundo en que se encuentra inmerso.

Ante las interrogantes ¿quiénes han tenido acceso al conocimiento? y ¿cuál es el conocimiento legitimado? cabe responder que los hombres, en este mundo patriarcal, han tenido mayores oportunidades para acceder al conocimiento, a los centros de estudio y la educación. En cuanto a la segunda, el conocimiento legitimado ha sido el científico, pero preferiblemente producido por hombres. Yadira Calvo, ha mostrado vastos ejemplos sobre qué ha pasado con las producciones intelectuales realizadas por mujeres. Con esto puedo afirmar que la epistemología dominante tiene fuertes matices androcéntricos.

Ahora bien, una vez que he aclarado lo anterior me adentraré en identificar las principales contribuciones teóricas que ha realizado la pensadora Gloria Comesaña Santalices para el proceso de construcción de una/s epistemología/s feminista/s. Si bien, la explicación previa para caer en el tema central del presente ensayo es extensa, considero que este preámbulo es necesario para tener presente el contexto en que surge la propuesta de esta autora, a partir de ello lograr una mayor comprensión de sus planteamientos y valorar la importancia de los mismos.

No es un secreto para nadie que la filosofía históricamente ha sido una disciplina androcéntrica, incluso la mayoría de filósofos reconocidos, por no decir todos, que son estudiados en las academias o reconocidos más allá de ellas son hombres. Esto no quiere decir que no existan mujeres filósofas, como al parecer la historia oficial ha intentado hacer creer. 
En este contexto, donde se entrecruzan diversos sistemas de dominación (patriarcado, modernidad, colonialismo, capitalismo), como lo proponen las feministas negras, que oprimen a diversos individuos, Gloria Comesaña Santalices decide reflexionar sobre la filosofía feminista, en específico, algunos temas claves de los que esta disciplina debe encargarse, entre ellos la epistemología feminista. Con estos trabajos esta filósofa pretende enriquecer mediante la crítica constructiva este proceso que ella misma afirma es "un ámbito del conocimiento que está todavía en pleno surgimiento, crecimiento y desarrollo" (Comesaña, 1998: p. 1).

Para realizar el presente ensayo he consultado tres de sus artículos: Aproximación a las características metodológicas de los Estudios de la Mujer, (1998); La ineludible metodología de género (2004) y Algunas reflexiones sobre la filosofía feminista (2007).

A continuación expondré diversos elementos constitutivos de la epistemología feminista que menciona la filósofa en estos textos.

En primera instancia considero importante mencionar, de manera breve, la distinción que Comesaña realiza entre feminismo filosófico y filosofía feminista. En cuanto a la primera alude que:

Consideramos que ciertamente hay un feminismo filosófico, el cual entendemos como la actividad de aquellas pensadoras feministas que aplican sus herramientas filosóficas a dilucidar todos o cualesquiera de los aspectos de la condición femenina en la Historia o en el mundo actual, con la idea de contribuir por esta vía de la toma de conciencia y la denuncia, a la transformación de dicha condición, que como hemos afirmado ya en numerosos escritos, es arbitraria y puede (y debe) ser cambiada." (Comesaña, 2007, p. 101).

Respecto a la segunda, afirma que:

Hay una filosofía feminista mas no femenina, es decir no con marcas de especificidad que vendrían de una supuesta esencia o naturaleza femenina que para nosotr@s no existe. La filosofía feminista es aquella hecha por mujeres (o varones) conscientes de la necesidad de un cambio en busca de la igualdad de derechos, el cual se fundamenta filosóficamente y no es sólo una demanda o aspiración "voluntarista", un deseo de justicia de "buenas" almas o quijotescas personalidades". (Comesaña, 2007, p. 101). 
La autora hace énfasis en que esta filosofía cuestiona a la filosofía oficial, androcéntrica, sexista...

En cada una de las descripciones y análisis que esta filósofa desarrolla aclara cuáles son o podrían ser algunas de las aristas de esta rama de la filosofía feminista, llamada epistemología feminista. En primera instancia mencionaré algunos de los aspectos que cuestiona esta rama de estudio:

- Oposición a las pretensiones de neutralidad/objetividad: Según Comesaña (2007) la epistemología feminista cuestiona la falsa neutralidad/objetividad que impone el conocimiento científico, debido a siempre van a mediar intereses, presupuestos, prejuicios, en sí, la subjetividad de la persona/s que están emprendiendo la investigación. La historia de la ciencia ha estado dominada por la razón no solo masculina, sino heterosexual, blanca, occidental... esto y el contexto han mediado las investigaciones que han realizado.

- Cuestionamiento a la pretensión de universalidad: Esta supuesta universalidad resulta de un movimiento ideológico que consiste en considerar al sujeto masculino (que es el "lugar" de donde pretendidamente parte la investigación), como si fuese asexuado, neutro, con lo cual los resultados obtenidos serían en efecto objetivos y universales, aplicables a todos los casos posibles. (Comesaña, 2007, p. 106).

A partir de esta pretensión, como lo señala la autora se ha impedido la intervención de otros sujetos, por ejemplos las mujeres, en la construcción del conocimiento.

- Relación sujeto-objeto. Según Comesaña (2007) desde la epistemología feminista se cuestiona la relación sujeto-objeto que impone el método de conocimiento dominante, ya que desde esta perspectiva se parte de un sujeto investigador activo, neutral/ objetivo, superior, que pretende investigar al objeto pasivo e inferior. Este sujeto toma a éste último como algo aislado al que pretende dominar para obtener la información necesaria, arrancarle sus secretos en función de sus intereses particulares. Desde el método científico sujeto-objeto no pueden implicarse, de lo contrario la investigación no tendrá resultados verídicos. La epistemología feminista, según la autora, no comparte esto, se 
opone a esta relación de verticalidad impuesta por el método de conocimiento dominante.

En sí podemos ver que la epistemología feminista cuestiona al conocimiento científico y la metodología utilizada por la ciencia; Comesaña Santalices (1988) al cuestionar estos aspectos, logra ser enfática en que la filosofía feminista:

Constituye un intento bastante logrado de ruptura epistemológica, que, en la medida en que sea profundizada, llevará al saber en general a renovarse y a adoptar una postura más auténtica y respetuosa, tanto del espíritu de cuestionamiento y búsqueda (sujeto), como de la alteridad hacia la que se dirige esta búsqueda (objeto) (p. 6).

¿Por qué una ruptura epistemológica?, podríamos preguntarnos. La filosofía feminista provoca esta ruptura epistemológica porque al posicionarse en contra de los parámetros que se han impuesto desde el conocimiento dominante propone metodologías alternativas. La autora comparte en sus artículos algunas de las características de esta metodología y que mencionaré brevemente

En primera instancia son conscientes de la falsa pretensión de neutralidad/ objetividad, ya que, todo conocimiento se encuentra situado, es decir, mediado por intereses políticos, sociales, económicos, culturales, sexuales... Ante esto la postura que toma la epistemología feminista es que: "Toda investigación debe pues asumir su propia posición subjetiva con respecto al objeto, y conocer, para controlarlos debidamente, los presupuestos de los que parte". (Comesaña, 1998: p.6). Para esta filósofa solamente cuando se asume la posición desde la que se parte a la hora de realizar una investigación, se podrán controlar los presupuestos, prejuicios que se poseen. Cabe señalar con ella que:

$\mathrm{Ni}$ el investigador ni la investigación son neutrales ni objetivos. A lo sumo, la objetividad, como una exigencia de respeto a la realidad del objeto, es una de las ideas reguladoras, una aspiración que debe guiar la actividad científica (1998, p. 5)

Otro de los aspectos que plantea esta rama de la filosofía feminista es el replanteamiento de la relación entre sujeto investigador/a- objeto investigado/a. Comesaña menciona que desde la Teoría Feminista no se parte de una relación vertical entre el sujeto-objeto, en la cual al sujeto no le es permitido involucrarse con el objeto de estudio. Ellas plantean una relación horizontal en la cual 
se establezca el acercamiento desde la propia realidad, identidad, circunstancia. No se parte de un sujeto activo, superior y un objeto pasivo, inferior, sino por el contrario sujeto y objeto coinciden y afirman-reconocen esta coincidencia.

La epistemología feminista parte de una dialéctica entre la relación sujeto-objeto, en la cual ambos convergen y se constituyen mutuamente. Por ello:

(...) aquí se agudiza la conciencia de los cambios que la investigación puede producir, no sólo sobre el objeto, lo cual es ya bastante importante, sino incluso sobre el sujeto, ya que en toda investigación, particularmente en la investigación sobre seres humanos, se da evidentemente una interacción entre sujeto y objeto en la cual, éste último incide sobre el sujeto transformándolo" (p. 5).

Dado esto, la relación sujeto-objeto sería sustituida por la relación sujeto-sujeto. Por eso, otro de los planteamiento fundamentales es considerar la diferenciación sexual como un fenómeno social y no como un fenómeno natural.

Comesaña (1998) plantea que es importante ser conscientes de que la diferenciación sexual, más que un fenómeno natural propiamente responde a una serie de interacciones históricas, sociales, políticas, económicas, que han valorado de manera positiva determinadas características (biológicas y sociales) y desvalorizado otras. En esta misma tesis, Vera Yamuni aporta en El ser y el valer de la mujer, escrito de 1985 .

La interdisciplinariedad en un doble sentido, es otro de los planteamientos. Según Comesaña (1989), con esto plantea, no solo enriquecer la teoría feminista a partir de la interacción de diversas disciplinas enfocadas en el estudio de la condición de la mujer, sino que también que las mismas autoras incursionen desde sus ámbitos del saber en otras disciplinas para que amplíen sus estudios sobre el tema en cuestión. Como último aspecto considero relevante retomar lo siguiente:

Está claro para las investigadoras que se ubican en el campo de los Estudios de la Mujer que la reflexión teórica no puede estar separada de la realidad social, y debe estar perentoriamente enraizada en la praxis cotidiana del quehacer de las mujeres y comunidades e incidir sobre el mismo (Comesaña, 1998, p. 15).

A modo de conclusión, considero que las contribuciones teóricas que aporta la filósofa Gloria Comesaña 
Santalies son sumamente pertinentes para este proceso de construcción en el que se encuentra tanto la filosofía feminista, como la misma epistemología que se plantea desde esta disciplina. En medio de este contexto las luchas que busquen reivindicar nuevos conocimientos, que busquen ampliar ese paradigma tan rígido que solo considera un único tipo de conocimiento, un único método como válido y un único sujeto como portador del mismo en detrimento de otros conocimientos y sujetos existentes, son muy valiosas e importantes, ya que, cuestionan el orden establecido dominante y no solo se quedan en la crítica sino que también plantean nuevas formas de abordar las cosas, de comprender estas realidades en que nos encontramos inmersos/as.

Desde mi campo de estudio, que es la filosofía, considero que estas propuestas deben ser incluidas en el programa de estudio de la carrera. El actual programa para obtener el bachillerato en filosofía tiene fuertes matices androcéntricos y occidentalocéntricos, debido a que no contempla el estudio de ninguna filósofa ni de la filosofía latinoamericana. A lo largo de la carrera se contemplan solamente las teorías propuestas por filósofos europeos. Estas que son importantes de estudiarse para tener un amplio panorama de la historia de la filosofía, pero debe hacerse de una manera equilibrada ya que actualmente con el diseño del pensum de estudios cualquier estudiante puede culminar la carrera pensando que nunca existió una mujer filósofa, ni filósofos latinoamericanos o que los aportes de unas y otros para el desarrollo de esta disciplina, han sido mínimos. Urge, desde mi punto de vista, que estas otras formas de conocimiento sean incorporadas en el programa de estudio, que sean legitimadas. Esta situación supone un desafío a resolver.

En cuanto a la viabilidad considero que al proyecto de la epistemología feminista aún le faltan cuestiones por afinar. Aún hace falta reforzar diversos aspectos, conceptos, categorías de análisis, es necesario organizar sistemáticamente las propuestas que se han desarrollado para que de esta forma puedan plantear metodologías más sólidas. Sin embargo, como la misma Comesaña lo menciona, este es "un ámbito del conocimiento que está todavía en pleno surgimiento, crecimiento y desarrollo" (Comesaña, 1998, p. 1). Ante esto, esfuerzos como el de esta filósofa son de gran importancia porque contribuyen al proceso de construcción crítica de estos proyectos que buscan, al fin y al cabo, visibilizar 
quienes que hemos sido invisibilizadas, desvalorizadas, deslegitimadas e inferiorizadas. Culmino el presente escrito con la siguiente cita: "Una de las cuestiones que aprendí del feminismo fue a sospechar de todo, dado que los paradigmas que se asumen en muchos ámbitos académicos están sustentados en visiones y lógicas masculinas, clasistas, racistas y sexistas". (Curiel, 2007, p. 93).

\section{Referencias bibliográficas}

Bóscan, A. (2010, Julio- Setiembre). "Gloria Comesaña Santalices: un nuevo rumbo programático para el movimiento feminista, desde una óptica latinoamericana". Utopía y praxis latinoamericana. Revista Internacional de Filosofía Iberoamericana y Teoría Social. Facultad de Ciencias Económicas y Sociales Universidad del Zulia Venezuela. 15(50):141150. Recuperado de xa.yimg.com/ kq/groups/16273644/1243131031/ name/Utopia15

Calvo, Yadira. (2004). Éxtasis y ortigas. Las mujeres entre el goce y la censura. Costa Rica: FARBEN, Grupo Editorial Norma.

Comesaña, G. (2007, Enero-Junio). Algunas reflexiones sobre la filosofía feminista. Revista Venezolana de Estudios de la Mujer. 12(28): 99-125. Recuperado de http://saber.ucv.ve/ojs/index.php/ rev_vem/article/view/2182/2077
(2004, Enero- Junio, 2004). La ineludible metodología del género. Revista Venezolana de Ciencias Sociales. 8 (1): 1-16. Recuperado de webs.uvigo.es/pmayobre/.../la_ineludible_metodologia_de_genero.doc

(Diciembre, 1998). Aproximación a las características metodológicas de los Estudios de la Mujer. Frónesis, Revista de Filosofía Jurídica, Social y Política. 5 (3): 1-18. Recuperado de www.webs.uvigo.es/pmayobre/textos/gloria/metodologia_estu_ mujer.doc

(1995). Filosofía, feminismo y cambio social. Maracaibo, Venezuela: Ars Gráfica.

Curiel, O. (Abril, 2007). Crítica poscolonial desde las prácticas políticas del feminismo antirracista. Nómadas. Universidad Central de Colombia. 26: 92-101

De la Cruz, Sor Juana Inés. (1998). Respuesta a Sor Filotea de la Cruz. México: Distribuidores Fontanera.

Gargallo, Francesca. (1997). Las ideas feministas latinoamericanas. Recuperado de http://webs.uvigo.es/pmayobre/ descargar_libros/las\%20ideas\%20 feministas\%20latinoamericanas.pdf

González, A. (1989). Introducción a la práctica de la filosofía. San Salvador, El Salvador: UCA Editores.

Guzamán, M. y Pérez, A. (2005). Las Epistemologías Feministas y la Teoría de Género. Cuestionando su carga ideológica y política versus resolución de

106 Contribuciones teóricas para la construcción de epistemologías otras no androcéntricas: una aproximación al planteamiento feminista de Gloria Comesaña Santalices María Laura Stephen-Chaves 
problemas concretos de la investigación científica. Cinta moebio 22: 112 126. Recuperado de www.moebio. uchile.cl/22/guzman.htm

Lindberg, D. (2002). Inicios de la ciencia occidental. Antonio Beltrán, Trad. Barcelona. España: Paidós.

Prada, G. (noviembre, 2003). El pensamiento latinoamericano como categoría de análisis. En Monografía para la Universidad de La Serena, Chile.

Rincón, L. (2009). Bases histórico-filosóficas de la teoría feminista. Costa Rica: Universidad Nacional, Escuela de Filosofía.

Yamuni Tabush, Vera. (1985). "El ser y el valer de la mujer comparados con el ser y el valer del hombre". En La naturaleza femenina: Tercer Coloquio Nacional de Filosofía. México: Universidad Nacional Autónoma de México. 